\title{
Le déploiement de la formation à distance au sein des facultés de médecine dans le contexte de la crise sanitaire liée à la COVID-19: et après?
}

\author{
Distance education as medical schools' response to COVID-19 outbreak: \\ what comes next?
}

Dans de nombreux pays, la crise sanitaire liée à la COVID-19 a entraîné la fermeture des universités, confrontant ces dernières à un extraordinaire défi : assurer la continuité pédagogique en ayant massivement recours à la formation à distance (FAD) [1-3], auprès d'un public qui ne l'avait majoritairement ni choisie, ni vécue, ni anticipée. Cette situation inédite pourrait laisser penser que la FAD pratiquée pendant la crise correspond au «standard» de la FAD. Dans une vision politicoéconomique de l'éducation, les parties prenantes pourraient aussi être tentées de substituer durablement et largement la formation à distance à la formation en présence (parfois encore dénommée par le discutable anglicisme «présentiel ).

La FAD fait référence aux situations dans lesquelles les enseignants et les apprenants sont séparés et doivent s'appuyer sur des ressources électroniques pour continuer à enseigner et à apprendre, ce qui les contraint à changer de posture. Les étudiants deviennent des apprenants isolés, qui doivent faire preuve d'autonomie dans la gestion et dans l'organisation de leur formation, ce qui explique le taux élevé d'abandon [4]. Les enseignants doivent quant à eux adopter plusieurs rôles: le scénariste, qui rédige une histoire visant à modéliser et à planifier les étapes successives d'un parcours de formation; le médiateur de l'information, qui traduit, grâce aux ressources technologiques, les activités pédagogiques sous la forme de contenus accessibles grâce à des interfaces numériques; l'animateur, qui crée une communauté virtuelle d'apprentissage $[4,5]$. Pour être efficace, cette démarche doit s'appuyer sur des principes reconnus en pédagogie, parmi lesquels le fait d'associer les apprentissages à la résolution de problèmes, de favoriser l'engagement actif des étu- diants, d'encourager les interactions entre les pairs et avec les formateurs, et de s'efforcer de construire des activités d'évaluation ayant une forte authenticité [6]. La mise en pratique de ces principes implique que la FAD ne peut pas se résumer à mettre en ligne des diaporamas sonorisés ou des questions à choix multiple (QCM). Elle se révèle donc en réalité très coûteuse en ressources et en temps [4,6].

Aucune théorie de la cognition ne suggère que la FAD doive être privilégiée au détriment d'une autre modalité d'enseignement [6]. À vrai dire, il pourrait même paraitre incongru de chercher à se mettre à distance des étudiants lorsqu'ils sont à proximité et disponibles pour des interactions en présence. Si la phase aiguë de la crise sanitaire liée à la COVID-19 rend compréhensible et acceptable le déploiement d'une forme raisonnée de $\mathrm{FAD}$, en tant que stratégie adaptative, ce constat devrait encourager chaque université à: (1) porter un regard critique sur la capacité qu'elle a eue à mettre en œuvre, dans l'urgence, une FAD à large échelle, et sur les nécessaires transformations qui lui permettront d'optimiser la réponse qui sera apportée lors de prochaines crises ; (2) adopter une utilisation rationnelle et pertinente de la FAD, lorsque l'enseignement en présence est impossible, au service de formats spécifiques (comme les classes inversées) ou pour enrichir les dispositifs en présence, dans le cadre des méthodes mixtes [5]; (3) améliorer la formation des étudiants et des enseignants à l'usage des outils de la FAD.

Cette crise sanitaire est aussi une opportunité inédite d'interroger le manque de la présence de l'autre dans la formation et, par conséquent, la place incontournable de la présence et de la relation à l'autre pour soutenir les processus, complexes, d'enseignement de d'apprentissage. 


\section{Thierry PELACCIA ${ }^{1,2, *}$ \\ Anne DEMEESTER ${ }^{2,3}$ \\ Bernard CHARLIN ${ }^{2,4}$ \\ Jean-François DENEF ${ }^{5}$ \\ Rémi GAGNAYRE ${ }^{6}$ \\ Hubert MAISONNEUVE ${ }^{7,8}$ \\ Ahmed MOUSSA ${ }^{4}$ \\ Mathieu NENDAZ ${ }^{7}$}

${ }^{1}$ Centre de formation et de recherche en pédagogie des sciences de la santé (CFRPS), Faculté de médecine,

Université de Strasbourg, Strasbourg, France ${ }^{2}$ Société internationale francophone d'éducation médicale (SIFEM), Faculté de médecine, Université de Strasbourg,

Strasbourg, France

${ }^{3}$ Aix Marseille Université, Laboratoire Apprentissage, didactique, évaluation, formation (ADEF - UR 4671),

Marseille, France

${ }^{4}$ Centre de pédagogie appliquée aux sciences de la santé

(CPASS), Faculté de médecine, Université de Montréal,

Montréal, Canada

${ }^{5}$ Comité exécutif Formation pédagogique des enseignants,

Conférence internationale des doyens et des facultés de médecine d'expression française CIDMEF, Louvain, Belgique

${ }^{6}$ Laboratoire Éducation et pratiques de santé (LEPS UR 3412), Université Sorbonne-Paris-Nord, Bobigny, France

${ }^{7}$ Unité de recherche et de développement en éducation médicale (UDREM), Faculté de médecine, Université de Genève, Genève, Suisse
${ }^{8}$ Unité des internistes généralistes et pédiatres, Faculté de médecine, Université de Genève, Genève, Suisse

*Correspondance et offprints : Thierry PELACCIA, Faculté de médecine, Centre de formation et de recherche en pédagogie des sciences de la santé, 4 rue Kirschleger 67075 Strasbourg cedex. Mailto: pelaccia@unistra.fr.

\section{Références}

1. Rasmussen S, Sperling P, Poulsen MS, Emmersen J, Andersen S. Medical students for health-care staff shortages during the COVID-19 pandemic. Lancet 2020;395:e79-e80.

2. Representatives of the STARSurg Collaborative, EuroSurg Collaborative, and TASMAN Collaborative. Medical student involvement in the COVID-19 response. Lancet 2020;395: 1254 .

3. Mahase E. Covid-19: Portugal closes all medical schools after 31 cases confirmed in the country. BMJ 2020;368: m986.

4. Masters K, Ellaway RH, Topps D, Archibald D, Hogue RJ. Mobile technologies in medical education: AMEE Guide No. 105. Med Teach 2016;38:537-49.

5. Liu Q, Peng W, Zhang F, Hu R, Li Y, Yan W. The effectiveness of blended learning in health professions: systematic review and meta-analysis. J Med Internet Res 2016;18:e2.

6. Pelaccia T. Comment [mieux] former et évaluer les étudiants en médecine et en sciences de la santé ? Louvain-la-Neuve : De Boeck Supérieur, 2016. 